\title{
Butterwegge, Christoph/Rinke, Kuno (Hrsg.): Grundeinkommen kontrovers. Plädoyers für und gegen ein neues Sozialmodell, 260 S., Beltz, Weinheim 2018.
}

\author{
Josef Schmid \\ Online publiziert: 20. November 2020 \\ (C) Der/die Autor(en) 2020
}

Gegenwärtig wird über das bedingungslose Grundeinkommen (BGE) viel und kontrovers diskutiert. Die Debatte spannt weit und reicht von einer einfachen Ergänzung des etablierten sozialen Sicherungssystems zum alternativen Sozialmodell. Wie funktioniert dieses und was sind die Vorzüge beziehungsweise Nachteile eines solchen sozialpolitischen Konzeptes? Darauf versucht der vorliegende Band Antworten zu geben. In ihrer Einleitung bieten die Herausgeber eine Arbeitsdefinition: ,In seiner heutigen Form wäre das bedingungslose Grundeinkommen ein steuerfinanzierter Universaltransfer, den sämtliche (Wohn-)Bürger/innen zwecks Sicherstellung ihres Lebensunterhalts ohne Bedürftigkeitsprüfung und ohne Verpflichtung zur Erwerbsarbeit erhalten würden“ (S. 7).

Ein klassischer Begründungsstrang für das BGE folgt liberalen Traditionen, die Thomas Straubhaar in seinem Beitrag aufnimmt. Bekannt ist das Konzept der negativen Einkommensteuer von Milton Friedman. Darauf aufbauend schlägt Straubhaar ein Existenzminimum von 9000Euro (steuerlicher Grundfreibetrag) für einen Erwachsenen beziehungsweise für ein Kind von 7.428Euro pro Jahr vor. Dabei wird nicht zwischen Erwerbstätigen und Erwerbslosen oder Selbstständigen unterschieden. Alle wären gleich zu behandeln. Das Grundeinkommen ersetzt hier die bisherigen Steuerfreibeträge und alle Sozialtransfers. Die Effekte lassen sich für verschiedene Haushalte und Einkommen berechnen. Wer demnach weniger als 24.000 Euro verdient, zahlt keine Steuern, sondern erhält maximal 12.000Euro. Straubhaar schlussfolgert, dass das BGE ,radikal, gerecht, einfach und transparent“" sei und ,liberalen, egalitären und individualistischen" Prinzipien entspreche (S. 31).

Richard David Precht geht zwar ebenfalls vom Leitbild der freiheitlichen Gesellschaft aus, sieht jedoch das Ende der Lohn- und Erwerbsarbeit als Hintergrund und Ursache. Ihm zufolge soll das BGE ausreichend hoch sein - mindestens 1500Euro im Monat (S. 38). Dass das sehr viel Geld kostet - mehr als das Dreifache des Bundeshaushaltes - ist ihm klar, sei aber etwa über eine Mikrosteuer auf alle Finanztransaktionen machbar: „Von allen Fragen, die das Grundeinkommen aufwirft, ist das der Finanzierung mithin das kleinste Problem. Viel spannender sind die psy-

\footnotetext{
J. Schmid $(\bowtie)$

Eberhard Karls Universität Tübingen, Tübingen, Deutschland

E-Mail: josef.schmid@uni-tuebingen.de
} 
chologischen Fragen, denn hier geht es um das Menschenbild in Gegenwart und Zukunft" (S. 42). Für Precht geht es um eine ,,andere Gesellschaft“, in der Erwerbsarbeit die prägende Rolle verloren hat, weil es durch Digitalisierung und Automatisierung sehr viel weniger Jobs geben wird. In dieselbe Kerbe schlägt Philip Kovce, wenn er neben dem Bedeutungsverlust von Arbeit - und im Übrigen auch von Familie - darauf verweist, dass das BGE Selbstbestimmung fördere, den „Machtverlust klassischer Institutionen“ (S. 58) vorantreibe und das „,neoliberale Subjekt“ befreie. Das gelte ebenso im Hinblick auf die Geschlechter - so Ute Fischer in ihrem Beitrag zur Geschlechtergerechtigkeit.

Sascha Liebermann lotet aus, worum es geht und worum nicht. Dazu definiert er das BGE als Individualleistung, die grundsätzlich und dauerhaft - also nicht nachrangig oder bedarfsbezogen - sein und nicht mit anderen Einkommen verrechnet werden soll. Bei Michael Opielka heißt das dann „Garantismus“ (S. 83). Die Höhe des BGE ist für Liebermann deshalb besonders wichtig, weil daran zum einen die Möglichkeit, überhaupt nicht arbeiten zu müssen, hängt und zum anderen das Problem, was daraus finanziert werden muss oder welche öffentliche Infrastruktur noch vorgesehen ist.

Im zweiten Teil des Bandes kommen die Kritiker zu Wort. Heinz-J. Bontrup hält das BGE für „,eine ökonomisch skurrile Forderung“ (S. 114) und sieht nicht nur erhebliche Finanzierungsprobleme, sondern einen „Angriff auf das Sozialstaatsprinzip“ (S. 121), weil dessen Grundprinzipien verletzt würden. Stattdessen plädiert er für Arbeitszeitverkürzung und Umverteilung. Ähnlich argumentiert Ralf Krämer und ergänzt, dass das BGE ,faktisch der universelle Kombilohn als Lohnsubvention für die Unternehmen“ sei (S. 142). Und Daniel Kreutz warnt vor einer gefährlichen „Konsens-Illusion“ durch die Propagierung eines BGE, wo doch ,diametral entgegengesetzte Interessen mit sehr unterschiedlicher Machtausstattung im Spiel sind“ (S. 164).

Gerhard Bäcker arbeitet die Debatte auf, indem er seine Kritik auf das Prinzip der „Bedingungslosigkeit“ konzentriert und dessen wesentliche Elemente „Einkommensunabhängigkeit“ sowie „Freiheit von Erwerbsarbeit“ für ,schlichtweg nicht realisierbar“ (S. 176) hält. Auch er betont die „Mühen der Ebene“ kleinteiliger Sozialreformen anstelle einfacher Lösungen. Georg Vobruba bezeichnet das treffend als „Utopiefalle“ (S. 224). Rainer Roth interpretiert das BGE skeptisch im Lichte der Geschichte der Menschenrechte und sieht darin keine Alternative zur Lohnarbeit; im Gegenteil stabilisiert es seiner Ansicht nach sogar die „kapitalistischen Eigentums- und Produktionsverhältnisse“ (S. 186). Christoph Butterwegge beschäftigt sich vor allem mit den Tücken verschiedener Modelle des BGE und stellt keinen Lösungsbeitrag für die anstehenden Probleme fest, sondern eher ein liberales Politikmodell. Denn mittels des BGE könne man ,,weder die Kluft zwischen Arm und Reich schließen noch den Zusammenhalt der Gesellschaft stärken“ (S. 213).

Der dritte Teil des Buches beginnt mit einem Beitrag von Norbert Erdmann und Eberhard Dechmann über das Modellprojekt in Finnland, bei dem 2000 Arbeitslose ein BGE erhalten haben. Freilich hält Vobruba den Ertrag von Experimenten für begrenzt, weil die gesamtgesellschaftliche Ebene relevant ist. Kuno Rinke und Andreas Wüste wechseln in den Bereich der politischen Bildung (und gegebenenfalls Schule), plädieren für die Anwendung des „Kontroversitätsprinzips“ (S. 239) und 
loten die fachdidaktischen Perspektiven für den Unterricht aus. Am Ende folgt eine Übersicht über die deutschsprachige Literatur zum BGE (S. 256ff.) sowie kurze Informationen zu den Autor_innen.

Was bleibt, ist ein Band zu einem wichtigen und sehr kontroversen Thema mit dem Problem aller Sammelbände, nämlich das der Redundanz. Und mit Sascha Liebermann kann man festhalten: „Nun ist es keineswegs so, dass auf der einen Seite Befürworter/innen und auf der anderen Kritiker/innen stehen, sodass beide fein säuberlich auseinandergehalten werden könnten. Befürwortende wie kritische Stellungnahmen sind nicht selten widersprüchlich, Einwände richten sich bei genauerer Betrachtung häufig eher gegen den Status quo als gegen das Bedingungslose Grundeinkommen“ (S. 68). Schließlich hat auch Georg Vobruba recht, wenn er kritisch anmerkt: Die Forderung nach einem BGE ,hat etwas stark Entlastendes. Man bewegt sich auf der heilen Welt des Normativen“ (S. 234); vor allem, wenn keine saubere Gegenfinanzierung präsentiert wird. Umgekehrt gilt dasselbe freilich auch für den Neoliberalismus-Vorwurf, der allenthalben erhoben wird.

Funding Open Access funding enabled and organized by Projekt DEAL.

Open Access Dieser Artikel wird unter der Creative Commons Namensnennung 4.0 International Lizenz veröffentlicht, welche die Nutzung, Vervielfältigung, Bearbeitung, Verbreitung und Wiedergabe in jeglichem Medium und Format erlaubt, sofern Sie den/die ursprünglichen Autor(en) und die Quelle ordnungsgemäß nennen, einen Link zur Creative Commons Lizenz beifügen und angeben, ob Änderungen vorgenommen wurden.

Die in diesem Artikel enthaltenen Bilder und sonstiges Drittmaterial unterliegen ebenfalls der genannten Creative Commons Lizenz, sofern sich aus der Abbildungslegende nichts anderes ergibt. Sofern das betreffende Material nicht unter der genannten Creative Commons Lizenz steht und die betreffende Handlung nicht nach gesetzlichen Vorschriften erlaubt ist, ist für die oben aufgeführten Weiterverwendungen des Materials die Einwilligung des jeweiligen Rechteinhabers einzuholen.

Weitere Details zur Lizenz entnehmen Sie bitte der Lizenzinformation auf http://creativecommons.org/ licenses/by/4.0/deed.de. 